\title{
Giant Pyogenic Granuloma Gravidarum of the Hand
}

\author{
Khan ESa, Ariff MS ${ }^{a}$, Eshagroni A ${ }^{b}$, Haflah NHMc, Abdullah Sc, Sapuan Jc \\ a Department of Orthopaedics, Traumatology and Rehabilitation, Kulliyyah (Faculty) of Medicine, International \\ Islamic University Malaysia (IIUM), Malaysia. \\ ${ }^{b}$ Department of Pathology, Universiti Kebangsaan Malaysia Medical Centre (UKMMC), Malaysia. \\ 'Department of Orthopaedics and Traumatology, Universiti Kebangsaan Malaysia Medical Centre (UKMMC), \\ Malaysia
}

\begin{abstract}
We describe a case of a giant pyogenic granuloma affecting the left index finger of a 23-year-old pregnant lady requiring surgical excision. Pyogenic granuloma gravidarum is a benign hyperplastic lesion that commonly presents on oral mucosa, typically the gums, in approximately 5\% of pregnant women. Skin lesions over the fingers are an unusual site during pregnancy with only a few cases reported in the literature. The lesion was excised and a diagnosis of lobular capillary haemangioma was made based on histopathological evaluation. We delayed surgery until after parturition since recurrence is likely to be lower due to lower levels of circulating oestrogen and progesterone. The prognosis is usually excellent following resection. However, recurrences have been reported regardless of the treatment method.
\end{abstract}

KEYWORDS: Granuloma gravidarum, pregnancy tumour, pyogenic granuloma, hand tumour.

\section{INTRODUCTION}

The term pyogenic granuloma is a misnomer, and lobular capillary haemangioma is the currently preferred term. Epulis gravidarum manum is another proposed term to describe this lesion. ${ }^{1}$ Granuloma gravidarum usually appears in the first trimester and rapid growth accompanies the steady increase of circulating estrogens and progesterone. Management of granuloma gravidarum depends on the severity of the symptoms and signs. Surgery should be recommended if bleeding or pain from the lesion impedes routine daily activities, ${ }^{2}$ or after delivery, if the lesion has not regressed completely. ${ }^{1,3,4}$ Clinical observation and regular follow-up appointments during pregnancy are advised if the lesion is small in size, painless and free of bleeding. ${ }^{1,2}$ Pyogenic granuloma lesions have no malignant potential, but up to $15 \%$ may recur following excision. ${ }^{4,5}$ This report presents a case of granuloma gravidarum in a patient in the last trimester of pregnancy.

Corresponding author:

Dr. Mohd Ariff Sharifudin

Department of Orthopaedics, Traumatology and

Rehabilitation,

Kulliyyah (Faculty) of Medicine, International Islamic University Malaysia,

Jalan Hospital, 25150 Kuantan, Pahang, Malaysia

Tel: $\quad+60193352499$

Fax: $\quad+6095144451$

Email: ariffs@iium.edu.my/ mdariffs@hotmail.com

\section{CASE REPORT}

A 23-year-old lady, who is a right hand dominant and 18 days postpartum, presented with a painless solitary mass over her left ring finger for one month duration. The swelling was over the ulnar aspect of the distal phalanx (Figure 1a and 1b). It had progressively enlarged and bled frequently, both spontaneously and on trivial injury with a foul smelling serous discharge. The base of the mass was painful on motion.

She could not recall any significant trauma to the area prior to the development of the lesion. It began as a small nodule size of a pin head at 36 weeks of gestation. It was itchy and she scratched the lesion until it increased in size to a green pea. She sought treatment at a local hospital and was advised to wait until her delivery prior to any surgical intervention. During this period she went to a nearby clinic for daily dressing.

On one occasion, her nephew accidentally stepped on it, and it bled doubling its size the next day. There were no other swellings on her body, no history of fever and no constitutional symptoms. Examination revealed a bright red fungating mass about $3.0 \times 2.5$ $\mathrm{cm}$, well-circumscribed and ulcerated with a necrotic patch over the surface. The surrounding skin was inflamed suggestive of a secondary bacterial infection. The mass was soft to firm in consistency, non-tender on palpation and sensation was reduced over the ulnar side distal to the mass 

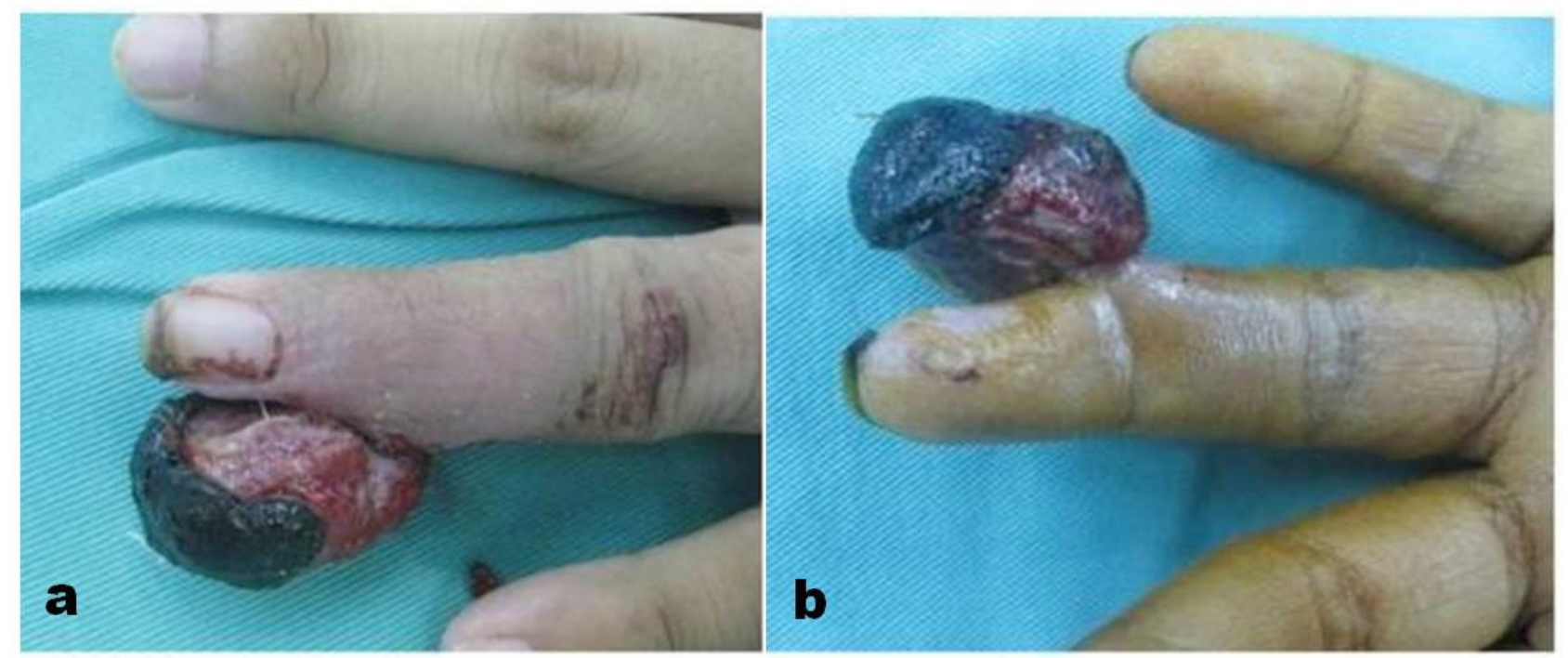

Figure 1. Illustration of the mass; a) view from dorsal aspect, and b) from volar aspect.

Movement of the interphalangeal joints of the affected finger were not restricted. There was no other skin, mucosal abnormalities or palpable lymph nodes. An inflammatory marker (CRP $3.4 \mathrm{mg} / \mathrm{dl}$ ) was elevated, suggesting a secondary bacterial infection.
She completed a one week course of cloxacillin. Radiographs did not show any bony erosion although the mass was radio-opaque suggestive of calcified material (Figure 2).

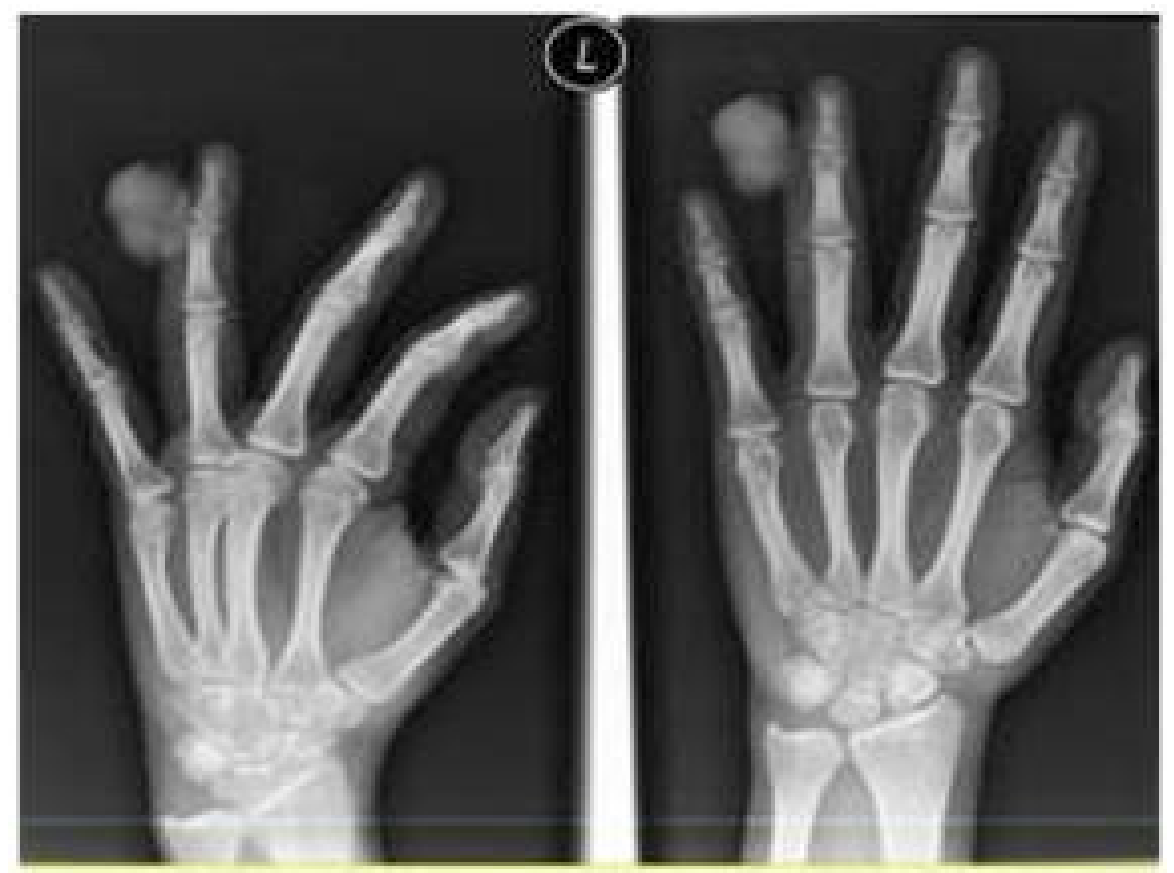

Figure 2. Hand radiographs showing a radiopaque mass without bony involvement.

The mass was clinically diagnosed as a pyogenic granuloma of pregnancy. At 22 days postpartum, she underwent an excision biopsy. Intraoperatively, a multilobulated, fungating mass arose from the ulnar side of the left ring finger, measuring $3.0 \times 2.5 \times 1.5 \mathrm{~cm}$ with a small area of necrotic patch over the surface. We also noted presence of a thin stalk from its base containing a feeding capillary (Figure 3 ). The digital artery was not involved. The lesion was carefully excised and sent for histopathological evaluation.

Microscopic examination revealed a polypoidal lesion composed of aggregates of numerous variable sized capillaries and venules arranged in lobular pattern and separated by fibrous septa (Figure 4a). There were erosions of the skin surface associated with dense neutrophilic infiltration and covered with scabs (Figure $4 b)$. The capillaries are lined by endothelial cells and the surrounding stroma is oedematous. The capillaries are compressed and the surrounding stroma displayed mixed inflammatory cells infiltration. All these findings support the diagnosis of a pyogenic granuloma. An immunohistochemical analysis denotes negative oestrogen and progesterone receptors. We followed her up for one year and she had good cosmesis and satisfactory wound healing without any recurrence. 


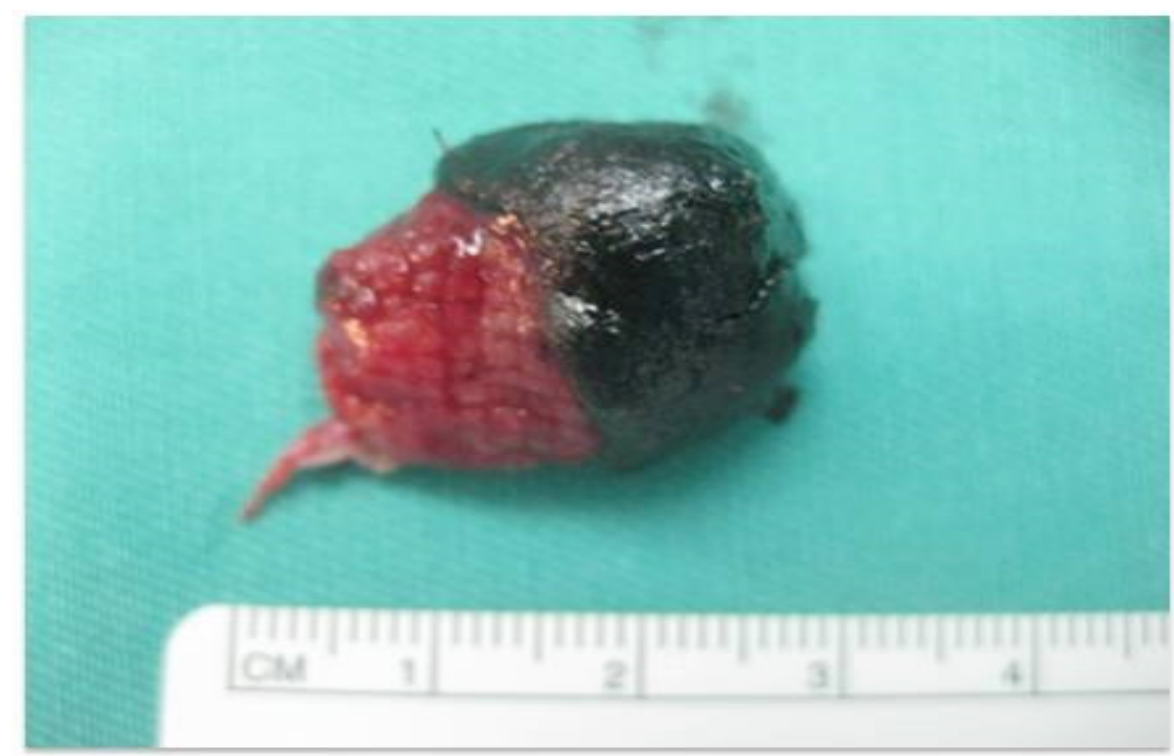

Figure 3. Gross specimen of the excised mass with the presence of granulation tissue over the base and necrotic tissue superiorly

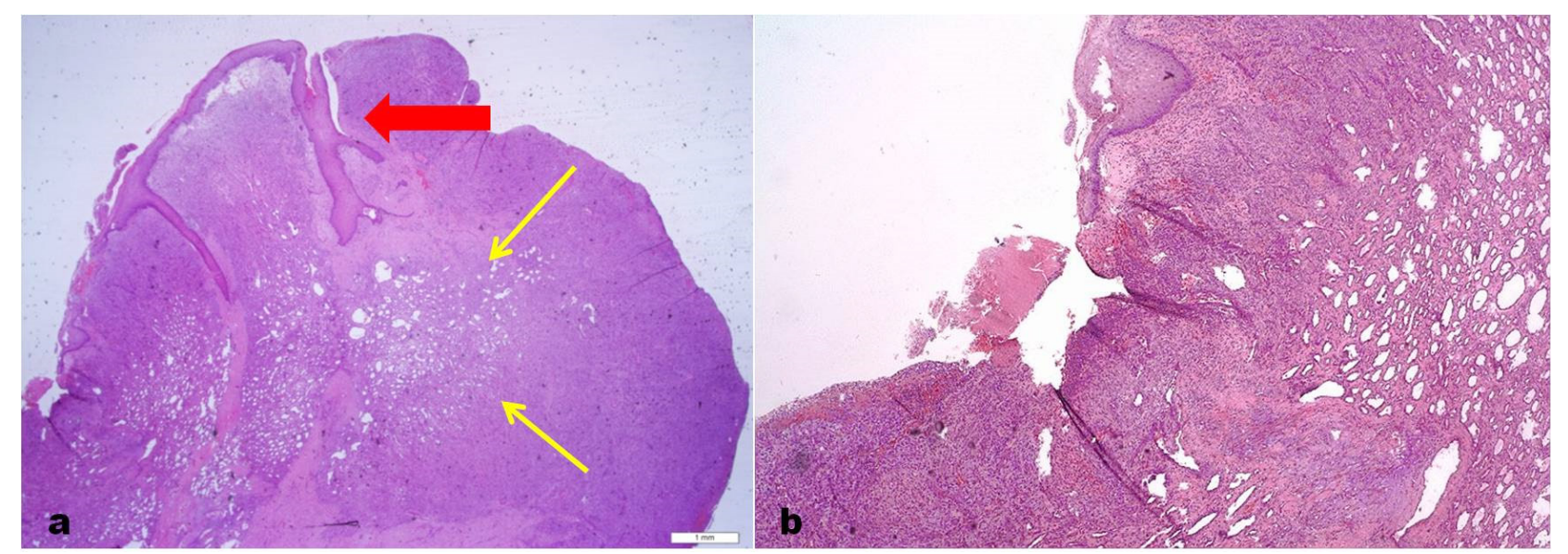

Figure 4. HPE sections revealed; a) a polypoidal lesion composed of aggregates of capillaries and venules (thin, yellow arrows) arranged in lobular pattern and separated by fibrous septa with surface ulceration (thick, red arrow), and b) erosion of the skin surface associated with dense inflammatory cells.

Microscopic examination revealed a polypoidal lesion composed of aggregates of numerous variable sized capillaries and venules arranged in lobular pattern and separated by fibrous septa (Figure 4a). There were erosions of the skin surface associated with dense neutrophilic infiltration and covered with scabs (Figure $4 \mathrm{~b})$. The capillaries are lined by endothelial cells and the surrounding stroma is oedematous. The capillaries are compressed and the surrounding stroma displayed mixed inflammatory cells infiltration. All these findings support the diagnosis of a pyogenic granuloma. An immunohistochemical analysis denotes negative oestrogen and progesterone receptors. We followed her up for one year and she had good cosmesis and satisfactory wound healing without any recurrence.

\section{DISCUSSION}

Pyogenic granuloma, otherwise known as acquired lobular capillary haemangioma, is a benign vascular tumour. These lesions are found primarily in the oral mucosa, but have been seen in the cutaneous aspect of the hand. ${ }^{2,3}$ They most often affect children and young adults, at times pregnant women and rarely, elderly individuals. ${ }^{1,4,6}$ The exact cause of pyogenic granuloma remains unknown. This name is inaccurate as it is not a true infection. Many have thought that it is caused by chronic or repetitive irritation or trauma, with subsequent infection resulting in a proliferative, vascular response to an infectious organism of low virulence. ${ }^{3}$ However, based on histological findings and the absence of trauma or irritation in some patients, others believe it is an acquired vascular lesion, possibly with secondary infection. The second hypothesis is similar to our patient's condition, although her traumatic event may have precipitated an increase in size.

Pyogenic granuloma has a strong association with pregnancy and usage of oral contraceptives, hence the name "pyogenic gravidarum". It usually appears in the first trimester and rapidly grows due to the steady increase of circulating oestrogens and progestins. ${ }^{2}$ In our patient, it started to develop during the end of 
her second trimester. Repeated trivial trauma and hormonal effect are sufficient to initiate the development of this lesion. Clinically, pyogenic granuloma must be differentiated from melanoma, particularly amelanotic melanoma, and other common differentials too, such as capillary haemangioma, angiosarcoma and squamous cell carcinoma. These entities were ruled out by both clinical findings and histopathology.

Grossly, the lesion is lobulated and haemorrhagic with ulcerating surface. Its bleeds easily and is usually found on the volar surface of the palm or digits. The lesions usually range from one millimetre to one centimetre in diameter but in this case it is much larger than usual. There is an initial period of rapid growth, which may be alarming and suspicious of a malignant cutaneous lesion. Histological examination demonstrates both granulation tissue and inflammatory cells. The histopathological examination revealed similar characteristics to those described by Sills et al.; loose granulation tissue, proliferation of endothelial cells, and abundance of capillary vessels, being typically accompanied by a mixture of infiltrated inflammatory cells. ${ }^{2}$ According to Ojanotko-Harri et al., there is no clinical or histological difference between pregnancy granuloma and pyogenic granuloma that occurs in nonpregnant patients. ${ }^{7}$

There are numerous options of treatment, including radiation, cauterization with silver nitrate, operative electrocautery, laser therapy, curettage, and excision performed with or without a wide margin. ${ }^{1-4,6,8,9}$ All of these approaches may be followed by recurrence. 4 Due to its gigantic size, we chose surgical excision as our method of treatment. This is more practical for such a big mass; cosmetically acceptable, and the rate of recurrence is less when done meticulously with the use of magnification, removal of a small cuff of normal tissue, and securing haemostasis. Treatment with antibiotics is usually unnecessary unless there is evidence of secondary bacterial infection. We believe that the recurrence rate is lower if removal is done after delivery since elevated levels of oestrogens during pregnancy may have an important role on the development of cutaneous lobular capillary haemangioma by direct hormonal action.

Mucosal and cutaneous pyogenic granulomas appear to be etiologically different, with a higher incidence of mucosal variant during pregnancy. Mucosal pyogenic granuloma has a higher female preponderance, and its relation to elevated levels of oestrogens during pregnancy has been demonstrated. ${ }^{8}$ However, some authors have suggested that elevated levels of oestrogens during pregnancy may also have an important role on the development of cutaneous lobular capillary haemangioma by direct hormonal action. ${ }^{9}$ Cutaneous pyogenic granuloma during pregnancy is not necessarily related to hormonal effect but we are unable to rule out the possibility even with negative oestrogen and progesterone receptor in our patient. It is still possible that oestrogen receptor or progesterone receptor were present in the lesion, but either the concentrations were too low or the antibodies and reagents used were not sensitive enough to identify them. ${ }^{10}$

\section{CONCLUSION}

We present a common lesion occurring in an uncommon site of the body during pregnancy. The history of rapid growth, tendency to bleed, and the pedunculated nature of the lesions are typical of pyogenic granuloma. Delaying the surgical removal until after parturition is advisable; recurrence rate is likely to be low due to lower levels of circulating oestrogen and progesterone. However, there remains the possibility of the mass enlarging during the pregnancy resulting in an unsightly lesion.

\section{REFERENCES}

1. Rader C, Piorkowski J, Bass DM, Babigian A. Epulis gravidarum manum: pyogenic granuloma of the hand occurring in pregnant women. J Hand Surg Am 2008; 33:263-5.

2. Sills ES, Zegarelli DJ, Hoschander MM, Strider WE. Clinical diagnosis and management of hormonally responsive oral pregnancy tumor (pyogenic granuloma). J Reprod Med 1996; 41:467-70.

3. Manus DA, Sherbert D, Jackson IT. Management considerations for the granuloma of pregnancy. Plast Reconstr Surg 1995; 95:1045-50.

4. Shall L, Stevens A, Saihan EM. Recurrent erythematous nodule on the sole of the foot. Recurrent pyogenic granuloma. Arch Dermatol 1991; 127:1219-20.

5. Kapadia SB, Heffner DK. Pitfalls in the histopathologic diagnosis of pyogenic granuloma. Eur Arch Otorhinolaryngol 1992; 249:195-200.

6. Mackie RM. Soft tissue tumors. In: Champion $\mathrm{RH}$, Burton JL, Burns DA, Breathnach SM, editors. Textbook of Dermatology. Blackwell Science: Oxford; 1998:2354-5.

7. Ojanotko-Harri AO, Harri MP, Hurttia HM, Sewon LA. Altered tissue metabolism of progesterone in pregnancy gingivitis and granuloma. J Clin Periodontol 1991; 18:262-66.

8. Yuan K, Wing LY, Lin MT. Pathogenetic roles of angiogenic factors in pyogenic granulomas in pregnancy are modulated by female sex hormones. J Periodontol 2002; 73:701-8.

9. Demir Y, Demir S, Aktepe F. Cutaneous lobular capillary hemangioma induced by pregnancy. J Cutan Pathol. 2004; 31:77-80.

10. Whitaker SB, Bouquot JE, Alimario AE, Whitaker TJ, Jr. Identification and semiquantification of estrogen and progesterone receptors in pyogenic granuloma of pregnancy. Oral Surg Oral Med Oral Pathol 1994; 78:755-60. 